\title{
Effects of Linderae radix extracts on a rat model of alcoholic liver injury
}

\author{
JUN-WEI WANG ${ }^{1}$, XIN-YI CHEN ${ }^{1}$, PEI-YANG HU ${ }^{1}$, MING-MING TAN ${ }^{1}$, \\ XIAO-GANG TANG ${ }^{1}$, MIN-CONG HUANG ${ }^{2}$ and ZHAO-HUAN LOU ${ }^{3}$ \\ ${ }^{1}$ Emergency Department, People's Hospital of Tiantai County, Tiantai, Zhejiang 317200; \\ ${ }^{2}$ Safety Evaluation Center, Zhejiang Academy of Medical Sciences, Hangzhou, Zhejiang 310007; \\ ${ }^{3}$ Institute of Material Medica, Zhejiang Chinese Medical University, Hangzhou, Zhejiang 310053, P.R. China
}

Received December 29, 2014; Accepted March 7, 2016

DOI: $10.3892 / \mathrm{etm} .2016 .3244$

\begin{abstract}
Traditional treatments have a poor effect on alcoholic liver diseases. Linderae radix (LR), the dried root of Lindera aggregata (Sims) Kosterm., has been frequently used in traditional Chinese medicine for treating various diseases, and has been shown to exhibit a protective effect on liver injury. In the present study, LR extracts were made using various solvents, and then administrated to rats to establish a model of ethanol-induced liver injury. The study aimed to investigate the therapeutic effects and potential mechanism of LR extracts on acute alcoholic liver injury. The serum levels of alanine aminotransferase (ALT), aspartate aminotransferase (AST), triglycercide (TG), cholesterol (TC), methane dicarboxylic aldehyde (MDA) and superoxide dismutase (SOD) were determined using an automatic biochemistry analyzer. In addition, pathological examination was performed by hematoxylin-eosin staining. The levels of MDA and SOD, and the expression levels of nuclear factor $(\mathrm{NF})-\kappa \mathrm{B}$, tumor necrosis factor (TNF)- $\alpha$ and interleukin (IL)- $1 \beta$ in liver tissue were investigated immunohistochemically. The expression of cytochrome P450 2E1 (CYP2E1) mRNA was quantified by reverse transcription-quantitative polymerase chain reaction. The results indicated that LR extracts improved the histopathological status and decreased the serum levels of ALT, AST, TG, TC and MDA. Furthermore, the levels of MDA and inflammatory mediators $(\mathrm{NF}-\kappa \mathrm{B}, \mathrm{TNF}-\alpha$ and IL-1 $\beta$ ) were decreased in liver tissues, and the overexpression of CYP2E1 mRNA induced by ethanol treatment. LR extracts exhibited a protective effect on alcoholic liver injury and the mechanism may be associated with the anti-inflammatory and anti-oxidative action.
\end{abstract}

Correspondence to: Dr Zhao-Huan Lou, Institute of Material Medica, Zhejiang Chinese Medical University, 548 Binwen Road, Hangzhou, Zhejiang 310053, P.R. China

E-mail: zhaohuanlouzhl@163.com

Key words: Linderae radix, acute alcoholic liver injury, anti-oxidant, anti-inflammatory

\section{Introduction}

Alcoholic liver disease (ALD) is the major cause of chronic liver disease, and can lead to fatty liver, hepatitis and cirrhosis $(1,2)$. In developed countries, ALD is a major cause of end-stage liver disease that requires treatment with liver transplantation (3). However, the scarcity of donor organs and complications associated with immunosuppression and transplant rejection limit the availability and clinical utility of liver transplantation (4). Traditional standard treatments of ALD, which include abstinence from alcohol, nutritional support and corticosteroid administration, have not been modified for at least 40 years, despite the poor outcomes of these methods $(3,5,6)$. Therefore, novel therapies are urgently required, particularly for patients with severe forms of ALD (such as alcoholic hepatitis), as well as for patients not achieving alcohol abstinence (3).

In recent decades, the use of herbal medicine in the prevention of ALD has attracted increased attention since it is multitargeted and presents less toxic effects (5,7). Linderae radix (LR; known as Wuyao in Chinese), the dried root of Lindera aggregata (Sims) Kosterm., has been frequently used in traditional Chinese medicine for treating various diseases, including abdominal pain and frequent urination $(8,9)$. LR consists of alkaloids, volatile oils and sesquiterpene esters, with alkaloids being the main active component $(8,10,11)$. Previous studies have found that total alkaloids in LR exert an anti-inflammatory effect by blocking the nuclear factor- $\kappa \mathrm{B}(\mathrm{NF}-\kappa \mathrm{B})$ and mitogen-activated protein kinase (MAPK) signaling pathways $(10,11)$. In addition, a study by Yamahara et al (12) revealed that the sesquiterpene in LR had a protective effect on liver injury induced by $\mathrm{CCl}_{4}$ or ethionine, and inhibited the increase in serum transaminase levels. A water extract of LR has been found to exert a cytoprotective activity against ethanol-induced acute gastric injury, and this protective activity was possibly mediated by endogenous prostaglandins and the vagus nerve (13). Certain previous studies have identified the active ingredients of LR and their pharmacological effects, such as the anti-inflammatory effect of alkaloids in the cardiovascular system and the anti-fatigue effect of flavonoids $(8-11,14)$; however, further research focusing on the protective effects of LR on the liver has rarely been reported. 
Therefore, in the present study, LR was extracted using various solvents in order to evaluate the therapeutic effects and potential mechanisms of LR extracts on alcoholic liver injury. These extracts were then administrated to a rat model of ethanol-induced liver injury that was established in the current study. The blood biochemistry and oxidation indices, as well as the mRNA expression levels of $N F-\kappa B$, tumor necrosis factor- $\alpha$ (TNF- $\alpha$ ), interleukin (IL) $-1 \beta$ and cytochrome P450 2E1 (CYP2E1), were determined to investigate the therapeutic potential and potential mechanisms of LR extracts on alcoholic liver injury.

\section{Materials and methods}

Animals. A total of 90 healthy male Sprague-Dawley (SD) rats (age, 6 weeks; weight, 170-200 g) were obtained from the Experimental Animal Center of Zhejiang Academy of Medical Sciences (Hangzhou, China). The rats were housed at $25 \pm 2{ }^{\circ} \mathrm{C}$ and exposed to a $12 / 12 \mathrm{~h}$ light-dark cycle, with free access to food and water. For $12 \mathrm{~h}$ before and $2 \mathrm{~h}$ after drug administration, the animals were fasted, but had free access to water. All experimental procedures were in accordance with the guidelines established by the Ethics Committee and Animal Management Committee of People's Hospital of Tiantai County (Tiantai, China).

Preparation of LR extracts using different solvents. Dried roots of Lindera aggregata (Sims) Kosterm. were provided by Zhejiang Biological Engineering Co., Ltd. (China). Dried root (200 g) extract was obtained by heating with water (dilution, $1: 12$ ) at $100^{\circ} \mathrm{C}$ for $1.5 \mathrm{~h}$, followed by leaching to obtain a decoction. The remaining dregs were extracted again by heating with 1:10 water at $100^{\circ} \mathrm{C}$ for a further $1 \mathrm{~h}$, and then leached. The decoction obtained during the two extraction processes was concentrated to $1 \mathrm{~g} / \mathrm{ml}$ under vacuum conditions and labeled as LR-water extract (LR-W). In addition, an LR-ethanol extract (LR-E) was obtained using a similar process, in which the same volume of $75 \%$ ethanol replaced water at room temperature and ethanol was removed under the reduced pressure conditions.

Subsequently, LR-E was successively extracted with petroleum ether, ethyl acetate and water-saturated butanol. Each extraction process was completed when the solution turned colorless, followed by collection and concentration of the extraction layer. The LR was divided into four components: Petroleum ether (LR-E1), ethyl acetate (LR-E2), butanol (LR-E3) and residual water (LR-E4). With the exception of LR-E1, the other extraction layers were directly diluted with water to obtain $1 \mathrm{ml}$ of solution equal to $1 \mathrm{~g}$ crude drug. LR-E1 was ground with addition of Tween-80 (Shanghai Dazhong Pharmaceutical Factory, Shanghai, China), mixed with water on a magnetic stirrer and diluted with water to obtain $1 \mathrm{ml}$ of solution corresponding to $1 \mathrm{~g}$ crude drug. The aforementioned solutions were diluted with water to a final concentration of $0.5 \mathrm{~g} / \mathrm{ml}$ and stored until further use.

Animal treatment. The 90 healthy male SD rats were randomly divided into nine groups ( $\mathrm{n}=10$ in each) as follows: Normal control, ALD model, positive control, LR-W, LR-E, LR-E1, LR-E2, LR-E3 and LR-E4 groups. All the rats were given the basal diet and water ad libitum. Rats in the normal control group were administrated with $1 \mathrm{ml}$ distilled water. Rats in other groups were intragastrically administrated with $1 \mathrm{ml}$ liquor (52\% ethanol; Beijing Red Star Co., Ltd., Beijing, China) per $100 \mathrm{~g}$ body weight (bw) in gradient concentrations (days 1-2, 30\% v/v; days 3-4, 40\% v/v; days 5-10, 50\% v/v), two times per day for 10 continuous days. After 10 days, an acute alcoholic liver injury model was established. As shown in Table I, the serum alanine aminotransferase (ALT) and aspartate aminotransferase (AST) of rats in model group were significantly higher compared with those in the normal control group $(\mathrm{P}<0.05)$, suggesting the successful establishment of the liver injury rat model.

During 10 days of liquor administration, rats in the normal control and model groups were fed daily with $1 \mathrm{ml} / 100 \mathrm{~g} \mathrm{bw}$ distilled water, while rats in the positive control were treated with $2.25 \mathrm{mg} / \mathrm{ml}$ dimethyl diphenyl bicarboxylate (DDB; Zhejiang Wanbang Pharmaceutical Co., Ltd., Taizhou, China), which is a widely used hepatoprotectant (15-17). Furthermore, rats in the LR treatment groups were orally administered with $1 \mathrm{ml} / 100 \mathrm{~g}$ bw LR extract once daily for 10 days. Subsequent to the last administration, blood samples were obtained from the inferior vena cava for biochemical assay. The rats were anesthetized and sacrificed using 5\% chloral hydrate (Shanghai Chemical Agent Cooperation, Shanghai, China), and liver tissue was immediately removed for oxidation index and histopathological analyses.

Blood biochemistry index assay. Blood obtained from the rats was centrifuged at $1,006 \mathrm{xg}$ for $15 \mathrm{~min}$ at room temperature to obtain serum. Next, the serum levels of ALT, AST, triglycercides (TG) and total cholesterol (TC) were measured using a TBA-120FR automatic biochemistry analyzer (Toshiba Medical System Co., Ltd., Tochigi, Japan), and according to the manufacturer instructions of the kits (Ningbo Meikang Biotechnology Co., Ltd., Ningbo, China). The details for the four kits were: ALT assay kit (cat. no. 20130517); AST assay kit (cat. no. 20130608); TC assay kit (cat. no. 20130626); and TG assay kit (cat. no. 20130719). Normal serum reference values for the analytes were as follows: ALT, 54.6-71.9 U/1; AST, 154.0-183.8 U/1; TC, 1.35-1.69 mmol/1; and TG, $0.27-0.38 \mathrm{mmol} / \mathrm{l}$.

Oxidation index determination. Liver homogenate was prepared by grinding $0.5 \mathrm{~g}$ liver tissue in $4.5 \mathrm{~g}$ physiological saline. The levels of malondialdehyde (MDA) and superoxide dismutase (SOD) in the serum and liver tissue were investigated using a PowerWave 340 microplate reader (Bio-Tek Instruments, Inc., Winooski, VT, USA), according to the manufacturer instructions of the MDA (cat. no. 20130819) and SOD (cat. no. 20130517) kits (Nanjing Jiancheng Bioengineering Institute, Nanjing, China). Normal serum reference values: MDA, 3.46-3.79 nmol/ml; SOD, 136.4-149.1 U/ml.

Histopathological examination. The left lobe of the liver was fixed with $10 \%$ neutral formalin, embedded in paraffin and stained with hematoxylin-eosin (Merck KGaA, Darmstadt, Germany). Tissue slides were observed under a B5-223IEP light microscope (Motic China Group Co., Ltd., Guangzhou, China) coupled with a Advanced 3.2 image analysis system (Motic China Group Co., Ltd., Xiamen, China). 
Table I. Effects of Linderae radix extracts on the blood biochemistry index of acute alcoholic liver injury rats.

\begin{tabular}{lccccc}
\hline Group & Dose $(\mathrm{g} / \mathrm{kg})$ & ALT $(\mathrm{U} / \mathrm{l})$ & AST (U/l) & TC (mmol/l) & TG (mmol/l) \\
\hline Normal control & - & $64.6 \pm 10.5$ & $165.2 \pm 20.0$ & $1.48 \pm 0.22$ & $0.326 \pm 0.063$ \\
Model & - & $87.9 \pm 29.0^{\mathrm{a}}$ & $191.1 \pm 16.8^{\mathrm{b}}$ & $1.85 \pm 0.42^{\mathrm{a}}$ & $0.453 \pm 0.093^{\mathrm{b}}$ \\
Positive control & 0.0225 & $70.3 \pm 8.6^{\mathrm{c}}$ & $174.3 \pm 12.4^{\mathrm{c}}$ & $1.39 \pm 0.19^{\mathrm{d}}$ & $0.523 \pm 0.122$ \\
LR-W & 2 & $75.6 \pm 16.3$ & $188.0 \pm 33.8$ & $1.53 \pm 0.14^{\mathrm{c}}$ & $0.418 \pm 0.121$ \\
LR-E & 2 & $61.2 \pm 10.2^{\mathrm{d}}$ & $166.9 \pm 13.0^{\mathrm{d}}$ & $1.51 \pm 0.37^{\mathrm{c}}$ & $0.361 \pm 0.106^{\mathrm{c}}$ \\
LR-E1 & 2 & $58.8 \pm 8.6^{\mathrm{d}}$ & $165.6 \pm 15.6^{\mathrm{d}}$ & $1.57 \pm 0.27^{\mathrm{c}}$ & $0.380 \pm 0.181$ \\
LR-E2 & 2 & $65.9 \pm 7.1^{\mathrm{c}}$ & $172.9 \pm 30.9$ & $1.63 \pm 0.36$ & $0.454 \pm 0.188$ \\
LR-E3 & 2 & $63.0 \pm 11.1^{\mathrm{c}}$ & $181.6 \pm 35.2$ & $1.69 \pm 0.27$ & $0.374 \pm 0.078^{\mathrm{c}}$ \\
LR-E4 & 2 & $69.3 \pm 13.0^{\mathrm{c}}$ & $174.2 \pm 31.6$ & $1.59 \pm 0.14^{\mathrm{c}}$ & $0.455 \pm 0.136$
\end{tabular}

${ }^{\mathrm{a}} \mathrm{P}<0.05$ and ${ }^{\mathrm{b}} \mathrm{P}<0.01$ vs. normal control group; ${ }^{\mathrm{C}} \mathrm{P}<0.05$ and ${ }^{\mathrm{d}} \mathrm{P}<0.01$ vs. model group. Positive control was treated with dimethyl diphenyl bicarboxylate. Linderae radix extracts obtained with the following solvents: Water (LR-W), ethanol (LR-E), petroleum ether (LR-E1), ethyl acetate (LR-E2), butanol (LR-E3) and residual water (LR-E4). ALT, alanine aminotransferase; AST, aspartate aminotransferase; TG, triglycercide; TC, cholesterol.

Table II. Sequences of primers used for reverse transcription-polymerase chain reaction.

\begin{tabular}{lll}
\hline Gene & Primer direction & \multicolumn{1}{c}{ Primer sequence } \\
\hline$\beta$-actin & Upstream & 5'-CATCATGAAGTGTGACGTTGAC-3' \\
& Downstream & 5'-TCAGGAGGAGCAATGATCTTGA-3' \\
& Probe & 5'-TGCTGTCTGGTGGCAC-3' \\
Cytochrome P450 2E1 & Upstream & 5'-GATATGTCATCCCCAAGGGTA-3' \\
& Downstream & 5'-CACACACACGCTTTCCTGC-3' \\
& Probe & 5'-CAGATCCAGAGAAGTTT-3' \\
\hline
\end{tabular}

Expression levels of $N F-\kappa B, T N F-\alpha$ and $I L-1 \beta$ in liver tissue. The expression levels of NF- $\kappa \mathrm{B}, \mathrm{TNF}-\alpha$ and IL- $1 \beta$ in liver tissue were detected using the labeled streptavidin-biotin method, according to the kit instructions (Santa Cruz Biotechnology, Inc., Santa Cruz, CA, USA). The monoclonal antibody catalogue numbers were as follows: Anti-mouse TNF- $\alpha$, (sc-8436); anti-mouse NF-кB; (sc-8436); and anti-rabbit IL-1 $\beta$, (sc-7884). Cells with yellow/brown particles were determined as having a positive expression.

Expression of CYP2E1 $\mathrm{mRNA}$ determined by reverse transcription-quantitative polymerase chain reaction $(R T-q P C R)$. The expression of CYP2E1 can be induced following ethanol consumption (18), thus this was determined in the present study. The total RNA in liver tissue was extracted using TRIzol reagent (Invitrogen; Thermo Fisher Scientific, Inc., Carlsbad, CA, USA). Next, total RNA was reverse transcribed into cDNA (RevertAid First Strand cDNA Synthesis kit; Thermo Fisher Scientific, Inc., Waltham, MA, USA). The mRNA expression of CYP2E1 in liver tissue was detected by qPCR coupled with a NanoDrop 2000 Spectrophotometer (Thermo Fisher Scientific, Inc.) and LightCycler 480 Instrument II (Roche Diagnostics, Basel, Switzerland). PCR was performed using a Fermentas First Strand cDNA Synthesis kit (cat. no. K1621; Thermo Fisher Scientific, Inc., Vilnius, Lithuania) and a Premix Ex Taq ${ }^{\mathrm{TM}}$ master mix kit (cat.no. RR390Q; Takara Bio, Inc., Tokyo, Japan), with $\beta$-actin used as an internal control. The primer sequences used in qPCR are shown in Table II. The PCR mixture consisted of $10 \mu 1$ Premix Ex Taq (Probe qPCR), $0.4 \mu 1$ forward primer, $0.4 \mu \mathrm{l}$ reverse primer, $1.5 \mu \mathrm{l} \mathrm{cDNA}, 0.4 \mu \mathrm{l} \mathrm{MGB}$ probe and $7.3 \mu \mathrm{l}$ nuclease-free water (total volume, $20 \mu \mathrm{l}$. The qPCR cycling conditions were as follows: $94^{\circ} \mathrm{C}$ for $30 \mathrm{sec}$, followed by 35 cycles at $94^{\circ} \mathrm{C}$ for $30 \mathrm{sec}, 62^{\circ} \mathrm{C}$ for $30 \mathrm{sec}, 72^{\circ} \mathrm{C}$ for $30 \mathrm{sec}$, and extension at $72^{\circ} \mathrm{C}$ for $7 \mathrm{~min}$. Subsequently, $10 \mathrm{ml} \mathrm{PCR}$ product was subjected to electrophoresis in a $1.5 \%$ agarose gel stained with ethidium bromide (Sigma-Aldrich, St. Louis, MO, USA).

Statistical analysis. All measurements are expressed as the mean \pm standard deviation, and SPSS 16.0 software (SPSS, Inc., Chicago, IL, USA) was used for statistical analysis. Values in different groups were compared with one-way analysis of variance, and the analysis between groups was performed with the least significant difference test. $\mathrm{P}<0.05$ was considered to indicate a statistically significant difference.

\section{Results}

Effect of LR extracts on serum enzyme levels. The levels of serum enzymes detected in the present study are presented in Table I. The serum levels of ALT and AST in the model group rats were significantly higher compared with those in the normal control group rats $(\mathrm{P}<0.05)$, suggesting the successful 
Table III. Effects of Linderae radix extracts on the serum oxidation index of acute alcoholic liver injury rats.

\begin{tabular}{|c|c|c|c|c|c|}
\hline Group & $\begin{array}{l}\text { Dose } \\
(\mathrm{g} / \mathrm{kg})\end{array}$ & $\begin{array}{c}\text { Liver MDA } \\
\text { (nmol/mg protein) }\end{array}$ & $\begin{array}{c}\text { Liver SOD } \\
\text { (U/mg protein) }\end{array}$ & $\begin{array}{l}\text { Serum MDA } \\
(\mathrm{nmol} / \mathrm{ml})\end{array}$ & $\begin{array}{c}\text { Serum SOD } \\
(\mathrm{U} / \mathrm{ml})\end{array}$ \\
\hline Normal control & - & $5.80 \pm 1.04$ & $194.7 \pm 20.4$ & $3.62 \pm 0.19$ & $143.5 \pm 7.5$ \\
\hline Model & - & $8.61 \pm 3.21^{\mathrm{a}}$ & $155.3 \pm 15.0^{\mathrm{b}}$ & $4.46 \pm 0.30^{\mathrm{b}}$ & $126.2 \pm 6.6^{\mathrm{b}}$ \\
\hline Positive control & 0.0225 & $7.66 \pm 3.38$ & $169.0 \pm 12.2^{\mathrm{c}}$ & $4.29 \pm 1.02$ & $138.4 \pm 10.3^{\mathrm{d}}$ \\
\hline LR-W & 2 & $6.57 \pm 3.17$ & $162.9 \pm 7.80$ & $4.46 \pm 0.59$ & $138.1 \pm 4.7^{\mathrm{d}}$ \\
\hline LR-E & 2 & $7.75 \pm 4.13$ & $165.7 \pm 12.8$ & $3.87 \pm 0.44^{\mathrm{d}}$ & $136.3 \pm 10.4^{\mathrm{c}}$ \\
\hline LR-E1 & 2 & $7.88 \pm 3.26$ & $167.7 \pm 10.0^{\mathrm{c}}$ & $4.50 \pm 0.64$ & $127.3 \pm 9.6$ \\
\hline LR-E2 & 2 & $7.26 \pm 3.68$ & $160.1 \pm 12.5$ & $4.50 \pm 1.22$ & $125.8 \pm 5.8$ \\
\hline LR-E3 & 2 & $7.98 \pm 2.65$ & $169.3 \pm 10.0^{\mathrm{c}}$ & $4.22 \pm 0.80$ & $133.3 \pm 10.1^{\mathrm{c}}$ \\
\hline LR-E4 & 2 & $6.96 \pm 2.07$ & $167.1 \pm 13.1^{\mathrm{c}}$ & $3.78 \pm 0.58^{d}$ & $140.2 \pm 6.4^{\mathrm{d}}$ \\
\hline
\end{tabular}

${ }^{a} \mathrm{P}<0.05$ and ${ }^{\mathrm{b}} \mathrm{P}<0.01$ vs. normal control group; ${ }^{\mathrm{P}}<0.05$ and ${ }^{\mathrm{d}} \mathrm{P}<0.01$ vs. model group. Positive control was treated with dimethyl diphenyl bicarboxylate. Linderae radix extracts were obtained with the following solvents: Water (LR-W), ethanol (LR-E), petroleum ether (LR-E1), ethyl acetate (LR-E2), butanol (LR-E3) and residual water (LR-E4). MDA, methane dicarboxylic aldehyde; SOD, superoxide dismutase.

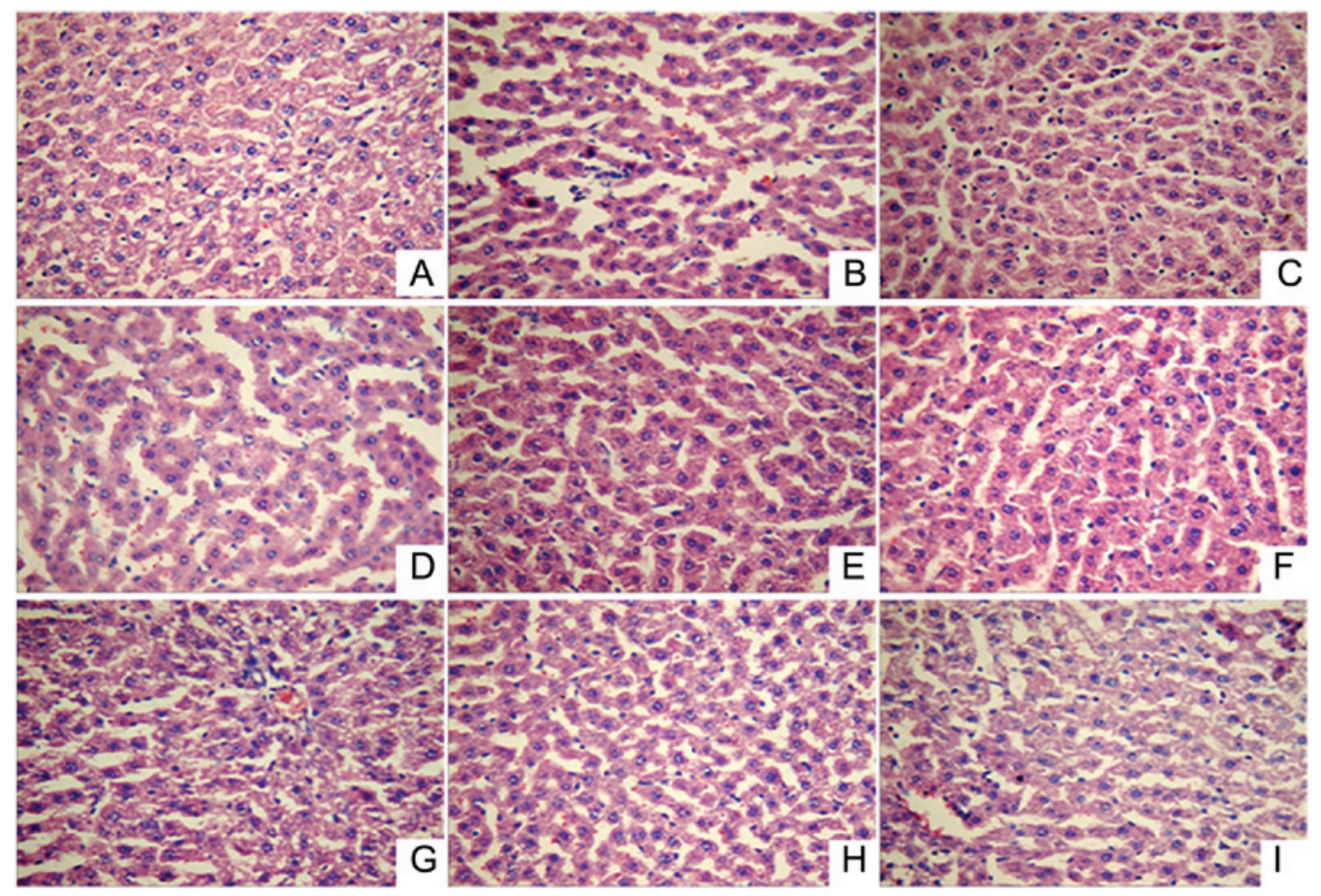

Figure 1. Liver tissue pathology (hematoxylin-eosin staining; magnification, $\mathrm{x} 400$ ) in the following extract groups: (A) Normal; (B) model; (C) positive control (dimethyl diphenyl bicarboxylate); and Linderae radix extracts with solvents including (D) water (LR-W), (E) ethanol (LR-E), (F) petroleum ether (LR-E1), (G) ethyl acetate (LR-E2), (H) butanol (LR-E3) and (I) residual water (LR-E4).

establishment of the liver injury rat model. Compared with the model group, serum ALT levels in the positive control, LR-E $(\mathrm{P}<0.01)$, LR-E1 $(\mathrm{P}<0.01)$, LR-E2 $(\mathrm{P}<0.05)$, LR-E3 $(\mathrm{P}<0.05)$ and LR-E4 $(\mathrm{P}<0.05)$ groups were significantly lower. In addition, serum AST levels in the positive control, LR-E and LR-E1 groups were significantly lower $(\mathrm{P}<0.05)$ compared with those in the model group. The results suggest that LR extracts suppress the increases of serum AST and ALT induced by alcoholic liver injury.

Effect of LR extracts on serum lipid parameters. The results of serum lipid parameter levels (TC and TG) are shown in
Table I. TC and TG levels in the model control group were significantly higher when compared with those in the normal control group $(\mathrm{P}<0.05)$. Compared with the model group, serum TC levels were significantly lower in the positive control, LR-W, LR-E, LR-E1 and LR-E4 groups. Furthermore, the serum TG levels in LR-E and LR-E3 groups were significantly lower when compared with those in the model control group $(\mathrm{P}<0.05)$. These results suggest that $\mathrm{LR}$ extracts inhibit increases of serum TG induced by alcoholic liver injury.

Effect of LR extracts on oxidation indices. As shown in Table III, MDA levels in the serum and liver tissue of the 

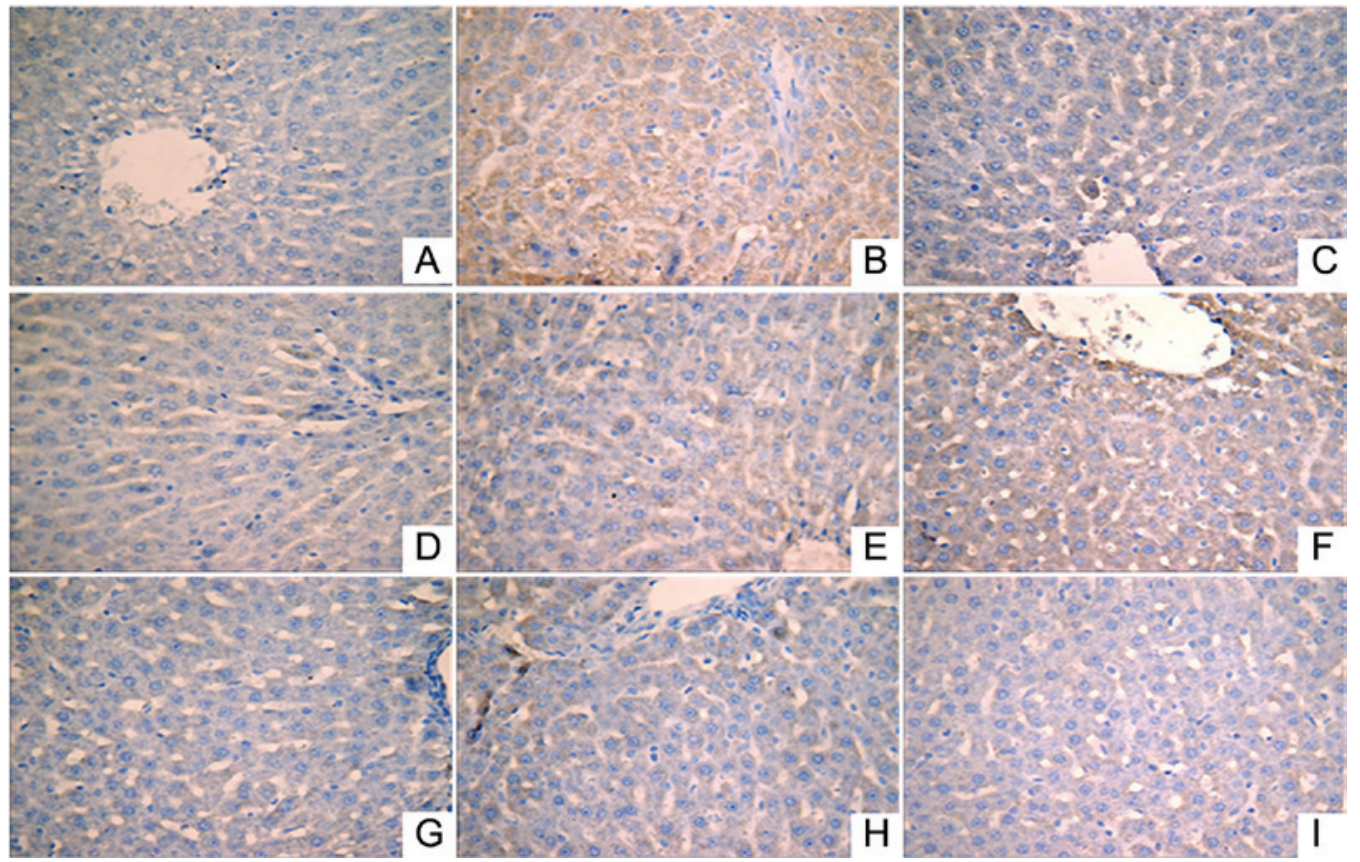

Figure 2. Expression of interleukin-1 $\beta$ in liver tissues (HRP-DAB $/ \mathrm{H}_{2} \mathrm{O}_{2}$ staining; magnification, $\mathrm{x} 400$ ) in the following extract groups: (A) Normal; (B) model; (C) positive control (dimethyl diphenyl bicarboxylate); and Linderae radix extracts with solvents including (D) water (LR-W), (E) ethanol (LR-E), (F) petroleum ether (LR-E1), (G) ethyl acetate (LR-E2), (H) butanol (LR-E3) and (I) residual water (LR-E4).

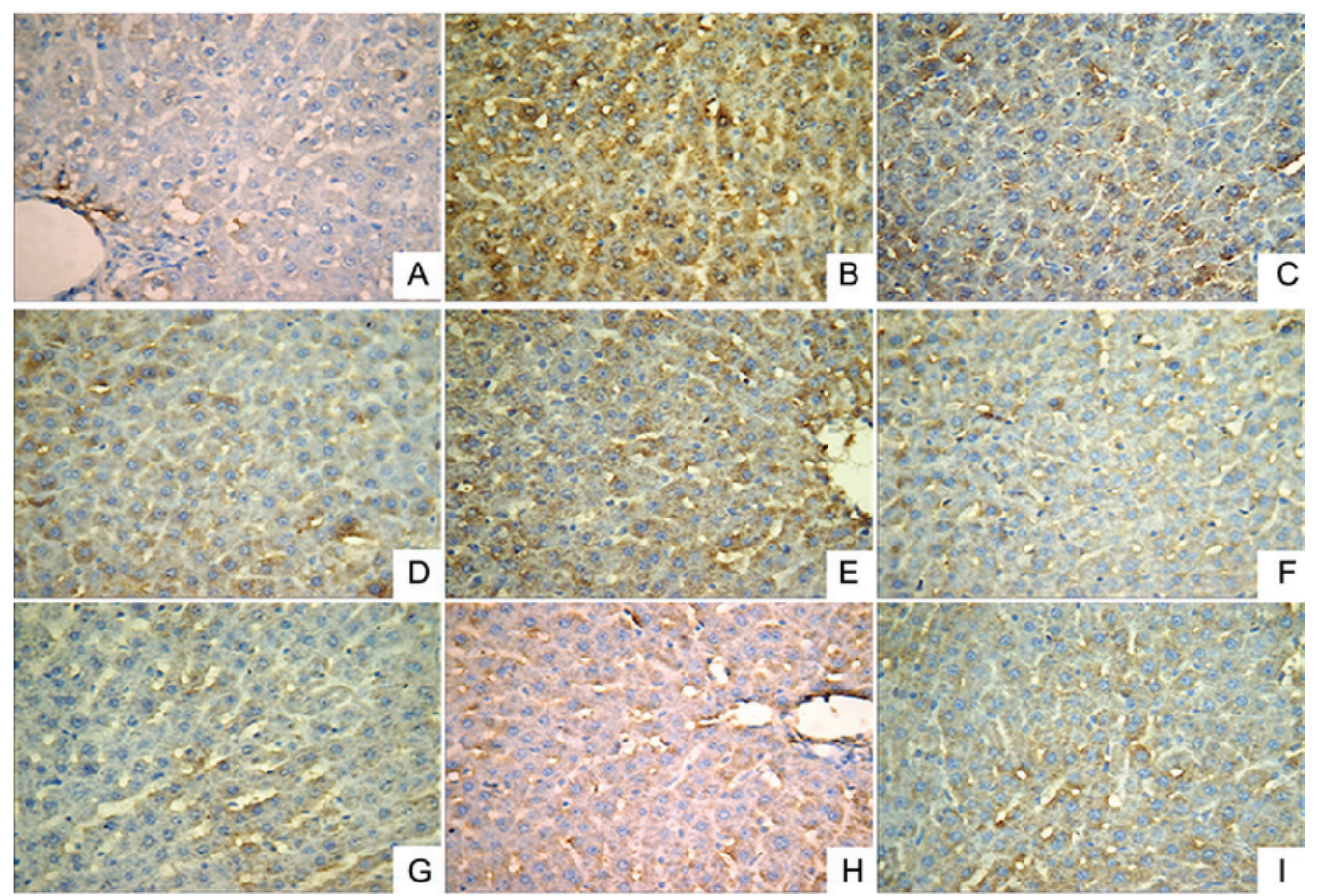

Figure 3. Expression of nuclear factor- $\mathrm{kB}$ in liver tissues (HRP-DAB $/ \mathrm{H}_{2} \mathrm{O}_{2}$ staining; magnification, $\mathrm{x} 400$ ) in the following extract groups: (A) Normal; (B) model; (C) positive control (dimethyl diphenyl bicarboxylate); and Linderae radix extracts with solvents including (D) water (LR-W), (E) ethanol (LR-E), (F) petroleum ether (LR-E1), (G) ethyl acetate (LR-E2), (H) butanol (LR-E3) and (I) residual water (LR-E4).

model control group was significantly higher compared with the normal control levels, while SOD levels were significantly lower $(\mathrm{P}<0.05)$. Compared with the model group, the serum MDA levels in the LR-E and LR-E4 groups were significantly decreased, while the serum SOD levels in the positive control, LR-W, LR-E, LR-E3 and LR-E4 groups were significantly increased $(\mathrm{P}<0.05)$. In addition, the liver
SOD levels in the positive control, LR-E1, LR-E3 and LR-E4 groups were significantly increased $(\mathrm{P}<0.05)$ compared with the model group; however, no statistically significant difference was observed in the liver MDA levels among the various treatment groups. These results indicate that LR extracts reduce alcoholic liver injury-related decreases in SOD levels. 


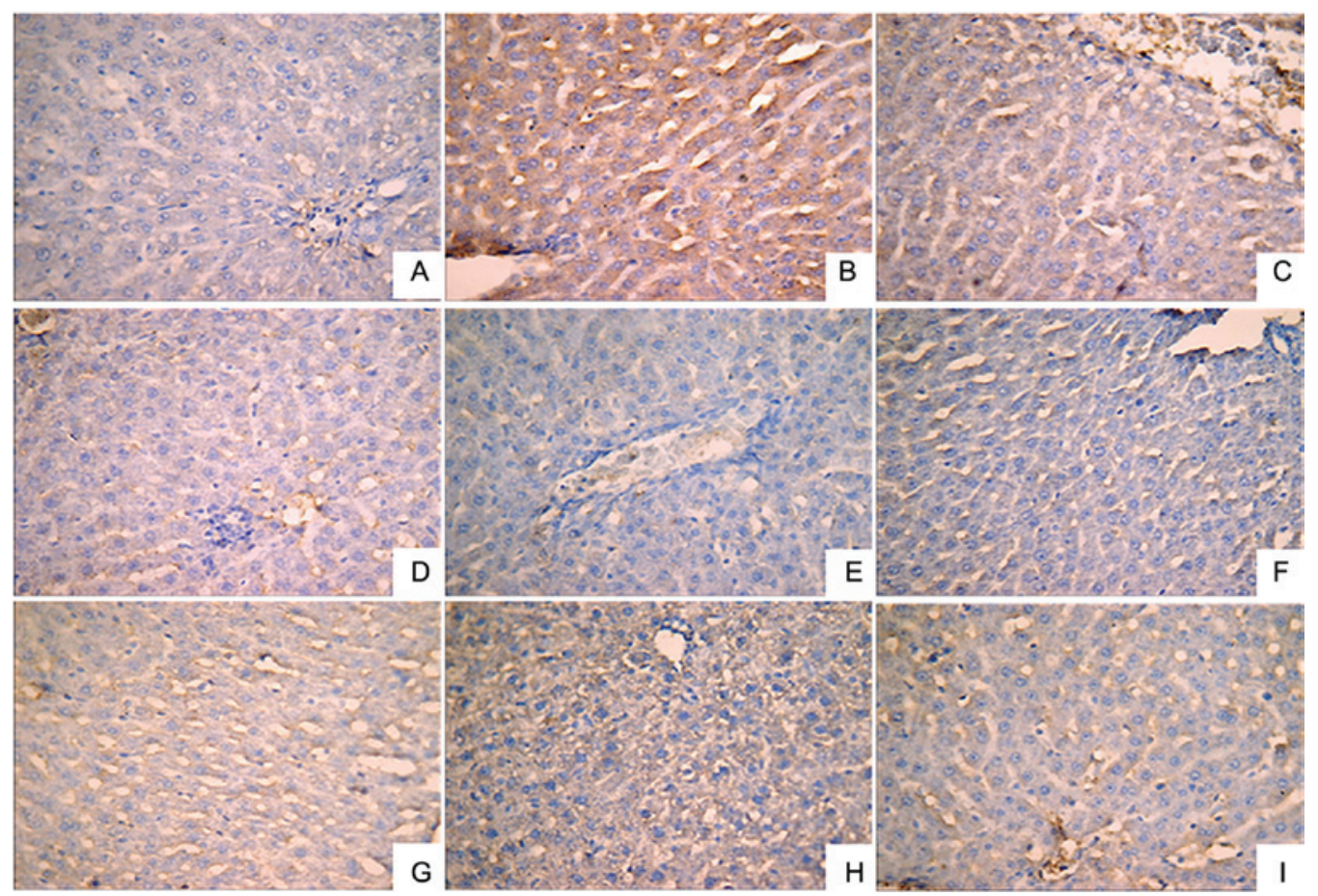

Figure 4. Expression of tumor necrosis factor- $\alpha$ in liver tissues (HRP-DAB $/ \mathrm{H}_{2} \mathrm{O}_{2}$ staining; magnification, $\mathrm{x} 400$ ) in the following extract groups: (A) Normal; (B) model; (C) positive control (dimethyl diphenyl bicarboxylate); and Linderae radix extracts with solvents including (D) water (LR-W), (E) ethanol (LR-E),

(F) petroleum ether (LR-E1), (G) ethyl acetate (LR-E2), (H) butanol (LR-E3) and (I) residual water (LR-E4).

Histopathological changes in liver tissue of ALD model rats. Visual inspection of the liver tissue obtained from the ALD rats revealed that the tissues were of normal color, the liver capsule was shiny, and no adhesion was identified between abdominal intestinal canals in the normal control group. By contrast, the liver color in the ALD model group rats was slightly yellow. The liver color in the various treatment groups was found to be similar to that in the normal control group.

Histopathological examination (Fig. 1) showed that the liver lobular structure was clear in the normal control group, with the hepatic cords arranged neatly by radiating from the central vein, while the liver cell outline was clear and polygonal. In addition, there was no hepatocellular necrosis, no significant pathological changes, and a small number of inflammatory cells was identified throughout the entire liver. However, in the acute alcoholic liver injury model rats, fuzzy hepatic lobule boundaries and scattered liver cells within the lobular in spotty necrosis were observed, the portal area showed inflammatory cell infiltration, certain cell nuclei had disappeared, and the liver cell cord was disordered. By contrast, in all the treatment groups (positive control and LR extract groups), the lobular boundaries were clear and hepatic cord cells were arranged neatly, while the inflammatory cell infiltration, necrosis and pathological changes in the liver tissue were significantly reduced. Thus, LR extract treatment appears to mitigate alcoholic liver injury.

Effect of LR extracts on the expression levels of $N F-\kappa B, T N F-\alpha$ and IL-1 $\beta$ in liver tissues. Immunohistochemical analysis was performed to investigate the expression levels of IL-1 $\beta$ (Fig. 2), NF- $\mathrm{KB}$ (Fig. 3) and TNF- $\alpha$ (Fig. 4). A small number of yellow/brown areas were observed in liver cells of rats in the

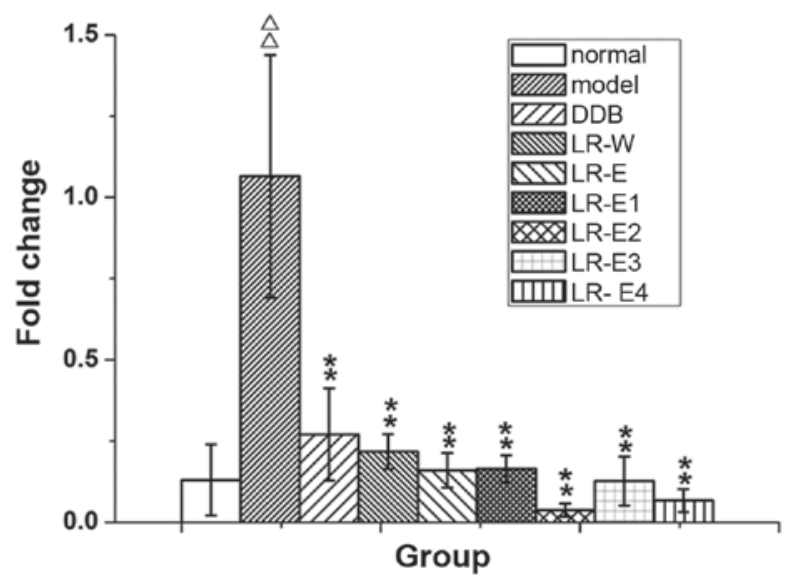

Figure 5. Expression of cytochrome P450 2E1 mRNA in liver tissues of rats. Linderae radix extracts were obtained with the following solvents: Water (LR-W), ethanol (LR-E), petroleum ether (LR-E1), ethyl acetate (LR-E2), butanol (LR-E3) and residual water (LR-E4). DDB, dimethyl diphenyl bicarboxylate. ${ }^{\Delta \Delta} \mathrm{P}<0.01$ vs. normal group. ${ }^{* *} \mathrm{P}<0.01$ vs. model group.

normal control group, suggesting low expression levels of IL-1 $\beta$, NF- $\kappa B$ and TNF- $\alpha$. Compared with the normal control group, the expression levels of IL- $1 \beta, N F-\kappa B$ and TNF- $\alpha$ in the model group were significantly increased. Treatment with DDB and the various LR extracts decreased the high expression levels induced by alcohol stimulation. These findings inidicate that LR extracts prohibit increases in the expression of NF- $\mathrm{KB}, \mathrm{TNF}-\alpha$ and IL-1 $\beta$ induced by alcohol consumption.

Effect of LR extracts on the expression of CYP2E1 $m R N A$ in liver tissues. The expression of CYP2E1 mRNA was detected by RT-qPCR (Fig. 5). Compared with the normal control group, 
CYP2E1 mRNA expression in liver tissue from rats in the model group was significantly enhanced $(\mathrm{P} \leq 0.01)$. Treatment with DDB and all LR extracts effectively reduced the expression of CYP2E1 mRNA that was induced by excessive alcohol intake $(\mathrm{P} \leq 0.01)$. The results indicate that $\mathrm{LR}$ extracts mitigate the increase of CYP2E1 detected in the alcoholic liver injury rat model.

\section{Discussion}

ALD is a common consequence of excessive alcohol abuse and remains a major cause of morbidity and mortality worldwide (19). LR has been widely used in traditional Chinese medicine, and extracts of LR have been reported to exert a therapeutic effect on various diseases, including abdominal pain and frequent urination $(20,21)$. In the present study, we successfully established an acute alcoholic liver injury model by gavage, and studied the preventive effect and the potential mechanisms of various LR extracts on the rat model. The results revealed that LR extracts had preventive effect on alcoholic liver injury by inhibiting serum ACT and AST levels, and this beneficial effect may be associated with anti-inflammation and anti-oxidation.

LR has been used in the treatment of various diseases (8), with certain studies reporting that LR extracts have a protective effect on ethanol-induced liver injury $(12,20,22)$. The activities of ALT and AST are commonly used as reliable markers for clinical monitoring of liver injury or liver function $(23,24)$. However, in the current study, the active component in each LR extract were not isolated and identified, which is a limitation of the study. Thus, further experiments isolating and identifying these active components in LR extracts may contribute to the development of novel treatments against ALD.

Inflammation serves a critical role in the pathogenesis and progression of ALD (7), while Kupffer cells play a key role in hepatic inflammation in liver injury (25). Excessive alcohol intake can activate Kupffer cells, resulting in the secretion of various inflammatory mediators, such as cytokines (TNF- $\alpha$ and IL-1 $\beta$ ) and NF- $\mathrm{B}$, which then further promotes inflammation and liver injury $(25,26)$. LR has been proven to exhibit anti-inflammation by preventing the production of inflammatory mediators $(10,27)$. Similarly, in the present study, LR extracts were found to inhibit the expression levels of $N F-\kappa B$, TNF- $\alpha$ and IL-1 $\beta$. Thus, this may suggest that LR extracts exhibit an anti-inflammatory effect on alcoholic liver injury by inhibiting the expression levels of NF- $\kappa \mathrm{B}, \mathrm{TNF}-\alpha$ and IL-1 $\beta$.

Ethanol-induced oxidative stress serves a significant role in the mechanisms underlying the development of ALD (28). CYP2E1 seems to be the main contributor to ethanol-induced oxidant stress (29). Expression of CYP2E1 has been shown to be significantly elevated upon exposure to ethanol, and CYP2E1 induces the production of reactive oxygen species (ROS), which are toxic to cells and result in lipid peroxidation (LPO) (29). MDA, the end-product of LPO, has been widely used as an indicator of LPO and a marker for the status of oxidative stress (30). Furthermore, SOD is essential for the endogenous anti-oxidative defense system to scavenge ROS and maintain the cellular redox balance (31). The RT-qPCR results showed that LR extracts may inhibit CYP2E1 expression in liver tissue, decrease the serum MDA levels, and elevate serum and liver tissue SOD activity to varying degrees. Thus, it is suggested that LR extracts may effectively protect the liver from ethanol-induced oxidative stress.

In conclusion, the results of the present study suggest that LR extracts exhibit a protective effect on alcoholic liver injury, and the mechanism may be attributed to the anti-inflammatory and anti-oxidative action. However, isolating and identifying the active components in LR extract is an indispensable step for the further clinical application of LR.

\section{Acknowledgements}

This study was supported by grants from the Project of Technology Application for Public Welfare in Zhejiang Province (grant no. 2013C33222), Medical and Health Project in Zhejiang Province (grant no. 2015DTA021), the Youth Project of National Natural Science Foundation (grant no. 81503328) and Project of Technology Application for Public Welfare in Zhejiang Province (grant no. 2016C33184).

\section{References}

1. Gao B and Bataller R: Alcoholic liver disease: Pathogenesis and new therapeutic targets. Gastroenterology 141: 1572-1585, 2011.

2. Xie YD, Feng B, Gao Y and Wei L: Characteristics of alcoholic liver disease and predictive factors for mortality of patients with alcoholic cirrhosis. Hepatobiliary Pancreat Dis Int 12: 594-601, 2013.

3. Altamirano $\mathrm{J}$ and Bataller R: Alcoholic liver disease: Pathogenesis and new targets for therapy. Nat Rev Gastroenterol Hepatol 8: 491-501, 2011.

4. Levine P, McDaniel K, Francis H, Kennedy L, Alpini G and Meng F: Molecular mechanisms of stem cell therapy in alcoholic liver disease. Dig Liver Dis 46: 391-397, 2014.

5. Woo GA and O'Brien C: Long-term management of alcoholic liver disease. Clin Liver Dis 16: 763-781, 2012.

6. Orman ES, Odena G and Bataller R: Alcoholic liver disease: Pathogenesis, management and novel targets for therapy. J Gastroenterol Hepatol 28 (Suppl 1): 77-84, 2013.

7. Ding RB, Tian K, He CW, Jiang Y, Wang YT, Wan JB and Huang LL: Herbal medicines for the prevention of alcoholic liver disease: A review. J Ethnopharmacol 144: 457-465, 2012.

8. Cheng XL, Ma SC, Wei F, Wang GL, Xiao XY and Lin RC: A new sesquiterpene isolated from Lindera aggregata (SIMS) Kosterm. Chem Pharm Bull (Tokyo) 55: 1390-1392, 2007.

9. Wu Y, Zheng Y, Liu X, Han Z, Ren Y, Gan L, Zhou C and Luan L: Separation and quantitative determination of sesquiterpene lactones in Lindera aggregata (Wu-yao) by ultra-performance LC-MS/MS. J Sep Sci 33: 1072-1078, 2010.

10. Luo Y, Liu M, Yao X, Xia Y, Dai Y, Chou G and Wang Z: Total alkaloids from Radix Linderae prevent the production of inflammatory mediators in lipopolysaccharide-stimulated RAW 264.7 cells by suppressing NF-kappaB and MAPKs activation. Cytokine 46: 104-110, 2009.

11. Wang C, Dai Y, Yang J, Chou G, Wang C and Wang Z: Treatment with total alkaloids from Radix Linderae reduces inflammation and joint destruction in type II collagen-induced model for rheumatoid arthritis. J Ethnopharmacol 111: 322-328, 2007.

12. Yamahara J, Matsuda H, Sawada T and Kushida H: Biologically active principles of crude drugs preventive effect of sesquiterpenoid components of the root of Lindera strychinifolia on experimental liver damage. Shōyakugaku Zasshi 37: 84-86, 1983 (In Japanese).

13. Zhu M, Luk CT and Lew TH: Cytoprotective Effect of Lindera aggregata roots against ethanol-induced acute gastric injury. Pharm Biol 36: 222-226, 1998.

14. Yi YN, Cheng XM, Liu LA, Hu GY, Wang ZT, Deng YD, Huang KL, Cai GX and Wang CH: Simultaneous determination of synephrine, arecoline and norisoboldine in Chinese patent medicine Si-Mo-Tang oral liquid preparation by strong cation exchange high performance liquid chromatography. Pharm Biol 50: 832-838, 2012. 
15. El-Beshbishy HA: The effect of dimethyl dimethoxy biphenyl dicarboxylate (DDB) against tamoxifen-induced liver injury in rats: DDB use is curative or protective. J Biochem Mol Biol 38 : 300-306, 2005.

16. Gao M, Zhang J and Liu G: Effect of diphenyl dimethyl bicarboxylate on concanavalin A-induced liver injury in mice. Liver Int 25: 904-912, 2005.

17. Abdel-Hameid NA: Protective role of dimethyl diphenyl bicarboxylate (DDB) against erythromycin induced hepatotoxicity in male rats. Toxicol In Vitro 21: 618-625, 2007.

18. Kessova I and Cederbaum AI: CYP2E1: Biochemistry, toxicology, regulation and function in ethanol-induced liver injury. Curr Mol Med 3: 509-518, 2003.

19. Albano E: Oxidative mechanisms in the pathogenesis of alcoholic liver disease. Mol Aspects Med 29: 9-16, 2008.

20. Ohno T, Takemura G, Murata I, Kagawa T, Akao S, Minatoguchi S, Fujiwara T and Fujiwara H: Water extract of the root of Lindera strychnifolia slows down the progression of diabetic nephropathy in db/db mice. Life Sci 77: 1391-1403, 2005.

21. Noda $Y$ and Mori A: Antioxidant activities of uyaku (Lindera strychnifolia) leaf extract: A natural extract used in traditional medicine. J Clin Biochem Nutr 41: 139-145, 2007.

22. Zhu M, Luk C and Lew T: Cytoprotective effect of Lindera aggregata roots against ethanol-induced acute gastric injury. Pharm Biol 36: 222-226, 1998.
23. Giannini EG, Testa R and Savarino V: Liver enzyme alteration: A guide for clinicians. CMAJ 172: 367-379, 2005.

24. Recknagel RO, Glende EA Jr, Dolak JA and Waller RL: Mechanisms of carbon tetrachloride toxicity. Pharmacol Ther 43: 139-154, 1989.

25. Luckey SW and Petersen DR: Activation of Kupffer cells during the course of carbon tetrachloride-induced liver injury and fibrosis in rats. Exp Mol Pathol 71: 226-240, 2001.

26. Thurman RG: II. Alcoholic liver injury involves activation of Kupffer cells by endotoxin. Am J Physiol 275: G605-G611, 1998.

27. Luo Y, Liu M, Xia Y, Dai Y, Chou G and Wang Z: Therapeutic effect of norisoboldine, an alkaloid isolated from Radix Linderae, on collagen-induced arthritis in mice. Phytomedicine 17: 726-731, 2010.

28. Das SK and Vasudevan DM: Alcohol-induced oxidative stress. Life Sci 81: 177-187, 2007.

29. Lu Y and Cederbaum AI: CYP2E1 and oxidative liver injury by alcohol. Free Radic Biol Med 44: 723-738, 2008.

30. Del Rio D, Stewart AJ and Pellegrini N: A review of recent studies on malondialdehyde as toxic molecule and biological marker of oxidative stress. Nutr Metab Cardiovasc Dis 15: 316-328, 2005.

31. Raychaudhuri SS and Deng XW: The role of superoxide dismutase in combating oxidative stress in higher plants. Botanical Review 66: 89-98, 2000. 